\title{
Binding of cationic pharmaceuticals to soil humic acids and the influence of inorganic cations
}

\author{
I. CHRISTL ${ }^{1 *}$ AND J.A. PEDERSEN ${ }^{2}$
}

${ }^{1}$ Institute of Biogeochemistry and Pollutant Dynamics, Department of Environmental Systems Science, ETH Zurich, 8092 Zurich, Switzerland

(*correspondance: iso.christl@env.ethz.ch)

${ }^{2}$ Departments of Soil Science, Civil \& Environmental Engineering, and Chemistry, Univ. of Wisconsin, Madison, WI 53706-1299, USA

(joelpedersen@wisc.edu)

\begin{abstract}
The increasing release of pharmaceuticals to the environment poses a serious long-term risk to ecosystem and human health. Among these organic pollutants, many compounds contain ionizable functional groups allowing them to form cationic species depending on the prevailing $\mathrm{pH}$ condition. Once released to the environment, natural organic matter (NOM) is considered to act as a main sorbent for these positively charged compounds. However, the ubiquitous presence of major cations such as e.g., $\mathrm{Ca}^{2+}$ and $\mathrm{Mg}^{2+}$ can alter the retention of cationic pharmaceuticals by NOM. In this presentation, the $\mathrm{pH}$ - and ionic strength-dependent binding of selected ionizable pharmaceuticals to soil humic acids as a representative of NOM will be shown to explain how the chemical properties of pharmaceuticals which are able to form cations relate to their retention by NOM. Examples will include the antibiotics clarithromycin and tetracycline as well as the anticonvulsant lamotrigine. In addition, experimental data and model predictions on competition between inorganic cations and ionizable pharmaceutical for binding to NOM will be presented to demonstrate the strong impact of major cations on the fate of ionizable pharmaceuticals in natural environments.
\end{abstract}

